Sharif University of Technology
Scientia Iranica
Transactions A: Civil Engineering
SCIENTIA
IRAN I CA

\title{
An algorithmic framework for improving the performance of the critical chain buffer sizing method
}

\author{
R. Ansaria,*, A. Makui ${ }^{\mathrm{b}}$, and P. Ghoddousi ${ }^{\mathrm{a}}$ \\ a. Department of Civil Engineering, Iran University of Science \& Technology, Narmak, Tehran, 16846, Iran. \\ b. Department of Industrial Engineering, Iran University of Science \& Technology, Narmak, Tehran, 16846, Iran. \\ Received 10 October 2015; received in revised form 4 August 2016; accepted 8 October 2016
}

\section{KEYWORDS \\ Buffer sizing; \\ Critical chain project \\ management; \\ Robustness; \\ Project cheduling; \\ Uncertainty.}

\begin{abstract}
Delays and disruptions are extremely challenging issues to deal with in project management. In this article, a novel optimization approach to the buffer sizing method is introduced aimed at maximizing the robustness of the buffered schedule generated. The measures affecting the buffer sizing include network complexity, flexibility, criticality, and robustness. The methodology presented is based on the critical chain project management concept, yet novel metrics are introduced to cover the uncertainties connected with the critical and non-critical chains. The overall purpose of the approach is to investigate the necessity and design of a decision support system to improve the process of critical chain project management. Utilizing a robust and flexible framework, this study tries to efficiently determine the size of feeding and project buffers. The weaknesses of the current critical chain project management approaches were overcome in the critical chain project management, and a new method was developed based on the integration of simulation and optimization techniques. In order to verify the efficiency of the method proposed, a case study is conducted. The outcomes indicate that the robust buffer allocation method proposed yields more stable project schedules, as against the traditional buffer sizing methods.
\end{abstract}

(C) 2018 Sharif University of Technology. All rights reserved.

\section{Introduction}

Designing reliable project plans is a complicated task. During the project execution phase, unforeseen events may disrupt the initial plan and cause resource conflicts. Hence, improving the robustness of the project schedule requires an advanced decision support system that accurately monitors the activities and the corresponding time buffers, predicts the potential resource conflicts, and reschedules tasks in a way that activity

\footnotetext{
*. Corresponding author.

E-mail addresses: raminansari@iust.ac.ir (R. Ansari); amakui@iust.ac.ir (A.Makui); ghoddousi@iust.ac.ir (P. Ghoddousi)
}

doi: $10.24200 /$ sci. 2017.4180 delays are minimized. Despite the scientific efforts devoted to developing sophisticated project schedules and control algorithms, few decision support systems exist to effectively plan and control the project status and reschedule activities during the execution, e.g. [15]. Existing project plan and control tools are capable of providing a new baseline plan through just manually updating the activity progress. Hence, the control and management of project activities during the execution phase is still mainly under the control of human experts who usually do not have access to accurate information about the future progress of activities, and the corrective actions chosen may be often suboptimal [6]. Based on these justifications, there is a crucial need for developing a more comprehensive and efficient decision support system to monitor the progress of activities and control the consumption of 
buffer times. Research in this area lets practitioners and academics do the robust planning of the project against future disruptions through the critical chainbased methodology. Considering the increasing uncertainty in project management, this research supports disruption recovery decisions to ensure effectiveness and efficiency. It is expected that the outcomes of this research will enhance the theory and practice of the project planning procedure as well as the project control process.

This research mainly aims at developing an innovative model for buffer sizing in order to improve the robustness of the project plan. The second objective is to design, utilize, and evaluate an advanced and robust project planning tool to support project managers in managing resource and time disruptions. To this end, some advanced procedures and methods have to be developed for the simulation and optimization of project plans. This research aims at developing an advanced planning tool based on Critical Chain Project Management (CC/PM) and optimization theory that acts in cases of disturbances as a decision support system to evaluate the impact of corrective actions on the performance of the project schedule. This task includes the development of a buffer sizing method as well as a buffer allocation model based on critical chain project management perspectives within a simulation framework. The main research question is "how can a simulation-based Decision Support System (DSS) support the CC/PM decision process?" This research is firstly aimed at identifying relevant and existing research approaches connected with $\mathrm{CC} / \mathrm{PM}$ for project management and investigating their similarities and differences, strengths, and weaknesses. Secondly, a novel approach is proposed that improves the performance of the traditional CC/PM methodology.

The article is outlined as follows. The literature review is provided in the next section. The methodology proposed is described in Section 3. In Section 4, the validation process of a benchmark case study is presented. The results and associated discussions are provided in Section 5. Finally, in Section 6, the conclusions are offered, and some recommendations for further researches are brought forth.

\section{Literature review}

Construction projects are subject to a wide range of limitations, uncertainties, and risks, including the complexity of the project, lack of knowledge of processes, and scarcity of resources. The purpose of project management is to ensure that the project will be implemented on time within budget and to guarantee the achievement of the predetermined objectives such as costs and optimal quality. The project planning and control processes are complex tasks that involve initial planning estimates based on available resources, measuring and evaluating activity progress, and corrective action requirements in terms of the unanticipated changes as well as deviations and uncertainties arising from disruptions [7]. The theory of disruption management refers to the dynamic correction of a baseline operational plan when disruptions occur. In the present study of disruption management, the researchers explore the proactive-reactive policies in both planning and execution phases. This research is also connected with the design of a decision support system to enhance the flexibility of the disruption management procedure. The topic of robust project planning has received growing scientific attention.

A taxonomy of the buffer sizing methods is given in Table 1. In the context of the Resource-Constrained Project Scheduling Problem (RCPSP), the disruption management field studies the problem of how to respond when an ongoing project is disrupted [8]. The goal is to get back on the track as soon as possible at the minimum deviation cost from the original schedule. To reduce such deviation costs, it is essential to take them into account when generating the new plan [9]. Typically, two different, yet interconnected, approaches are available to deal with disruptions. The first one consists of a proactive or robust approach that aims at generating stable project plans for different types of minor disturbances. The second approach is to take corrective actions such as rescheduling activities or reallocation of resources. These two main approaches to disruption management are intertwined; the researchers of this study aim at utilizing them in a decision support system.

The improvement of schedule stability and robustness is a concern in the management and control of projects, having attracted a lot of attention in recent researches [10]. The schedule robustness can be frequently reached through adding a percentage of the activity duration (safety time) to each activity so that in case of disruptions and without rescheduling, a percentage of project delays will be absorbed [11]. Time buffering is one of the most widely used approaches to robust project scheduling [12]. The use of time buffers prevents the spread of delays throughout the entire project network. The time buffering approach has two important decisions to be made. The first one involves the determination of the buffer sizes through considering the total costs (under the title of the buffer sizing problem). The second decision is to decide where to insert the time buffers in the project network (namely, the buffer allocation problem).

Critical Chain Project Management (CC/PM) is a well-known methodology of planning, controlling, and managing projects that puts emphasis on the resource constraints to perform project activities. This approach was originally introduced by Goldratt and 
Table 1. Literature classification of the current buffer sizing methods.

\begin{tabular}{|c|c|c|c|c|c|}
\hline Reference & $\begin{array}{l}\text { Buffer sizing } \\
\text { method }\end{array}$ & $\begin{array}{c}\text { Framework and } \\
\text { criteria }\end{array}$ & Approach & $\begin{array}{l}\text { Project } \\
\text { buffer }\end{array}$ & $\begin{array}{c}\text { Feeding } \\
\text { buffer }\end{array}$ \\
\hline Roel [42] & $\begin{array}{l}\text { Activity-Dependent } \\
\text { Float Factor (ADFF) }\end{array}$ & Float time & Heuristic & $\sqrt{ }$ & $\times$ \\
\hline \multirow[t]{2}{*}{ Tukel et al. [11] } & $\begin{array}{l}\text { Adaptive Procedure } \\
\text { with Resource } \\
\text { Tightness (APRT) }\end{array}$ & Resource tightness & Statistical & $\sqrt{ }$ & $\times$ \\
\hline & $\begin{array}{l}\text { Adaptive Procedure } \\
\text { with Density (APD) }\end{array}$ & Network density & Statistical & & \\
\hline Ma et al. [22] & $\begin{array}{l}\text { Flexible buffer } \\
\text { sizing approach }\end{array}$ & $\begin{array}{l}\text { Network density } \\
\text { Resource tightness } \\
\text { Network complexity } \\
\text { Risk preference }\end{array}$ & Statistical & $\sqrt{ }$ & $\sqrt{ }$ \\
\hline Bie et al. [43] & $\begin{array}{l}\text { Adaptive Procedure } \\
\text { With Activity } \\
\text { Dependence } \\
\text { (APAD) }\end{array}$ & $\begin{array}{l}\text { Dependence Degree (DD) } \\
\text { Dependence Factor (DF) }\end{array}$ & Statistical & $\sqrt{ }$ & $\times$ \\
\hline Zhang et al. [30] & $\begin{array}{l}\text { Buffer sizing based } \\
\text { on attribute } \\
\text { optimization }\end{array}$ & $\begin{array}{l}\text { Safety time } \\
\text { Resource tightness } \\
\text { Network complexity } \\
\text { Resource working efficiency } \\
\text { Resource cost index } \\
\text { Activity duration proportion } \\
\text { Activity cost index } \\
\text { Start time flexibility }\end{array}$ & Statistical & $\sqrt{ }$ & $x$ \\
\hline Ma et al. [28] & Improved CC/PM & $\begin{array}{l}\text { Environmental uncertainty } \\
\text { Activity complexity } \\
\text { Activity flexibility } \\
\text { Resource tightness } \\
\text { Risk preference }\end{array}$ & Statistical & $\sqrt{ }$ & $\sqrt{ }$ \\
\hline Ma et al. [29] & $\begin{array}{l}\text { Scenario-based } \\
\text { CC/PM }\end{array}$ & $\begin{array}{l}\text { Resource tension } \\
\text { Activity complexity } \\
\text { Environment uncertainty } \\
\text { Activity instability factor }\end{array}$ & $\begin{array}{l}\text { Scenario- } \\
\text { based } \\
\text { Stochastic } \\
\text { programming }\end{array}$ & $\sqrt{ }$ & $\times$ \\
\hline Bevilacqua et al. [31] & $\mathrm{CC} / \mathrm{PM}$ & - & $\begin{array}{l}\text { Goal } \\
\text { programming }\end{array}$ & $\sqrt{ }$ & $x$ \\
\hline Current study & $\begin{array}{l}\text { Robust Multi- } \\
\text { metric CC/PM }\end{array}$ & $\begin{array}{l}\text { Network complexity } \\
\text { Chain robustness } \\
\text { Chain flexibility } \\
\text { Noncritical chain dependency }\end{array}$ & $\begin{array}{l}\text { Simulation } \\
\text { and } \\
\text { optimization }\end{array}$ & $\sqrt{ }$ & $\sqrt{ }$ \\
\hline
\end{tabular}

employs the time buffers to protect the initial schedule against future disturbances [12]. Goldratt's critical chain scheduling and buffer management methodology are the practical applications of the Theory Of Constraints (TOC) to project management, extensively explored by the scholars. Fortunately, the theory of $\mathrm{CC} / \mathrm{PM}$ provides the opportunity for achieving an effective robust project schedule through managing time buffers. In this context, the critical chain is referred to as the series of both precedence and resource-dependent constraints that prevent a project from being finished in a shorter time, given the resource constraints. The CC/PM introduces three types of buffers including Feeding Buffer (FB), Project Buffer (PB), and Resource Buffer (RB). The project buffer or safety time is added after the last activity to protect the due-date performance. The feeding buffer is added where a non-critical chain connects with a critical chain [13].

The successful application of CC/PM depends on choosing the appropriate method of determining buffer sizes. The classic and well-known methods in the literature include the Cut and Paste Method (C\&PM), Root Square Error Method (RSEM), Adap- 
tive Procedure with Density (APD), and Adaptive Procedure with Resource Tightness (APRT). Van de Vonder et al. [14] addressed the setting of a balance between the quality and solution robustness, using the buffer allocation technique in a CC/PM framework. The quality robustness was measured in terms of the project duration, whereas the solution robustness refers to the deviation of the realized activity start time from the scheduled one. Most of the recent buffer sizing methods have developed the classic RSEM to size the project and feeding buffers through attribute optimization. Furthermore, special attention has been paid to the introduction of new measures that affect the size of the buffers. Tukel et al. [11] investigated buffer allocation methods already mentioned. RSEM uses two estimates for each activity including a safe estimate and an average one. It then computes the difference between safe and average approximations. The square root of the sum of squares of the difference for each activity is regarded as the buffer size [16]. Azaron and Modarres [17] proposed a constant-time Markov model in order to compute the project completion time distribution through a dynamic PERT network analysis. It was assumed that the activity durations follow a random distribution (e.g., Erlang or negative exponential).

The previous research suggested that different factors are important to be considered during the buffer sizing procedure. These factors are mostly characterized by complexity, flexibility, dependency, and robustness. As noticed by Tukel et al. [11], the network complexity is reflected as a ratio of the total number of precedence relationships to the total number. The activity dependency criteria were originally introduced by Leach [18] in the context of the CC/PM. Zhang et al. [19] proved that the codependency among project activity durations produced by the complexity has significant impact on buffer sizes. On this basis, the concepts of the rework safety time and rework probability matrix were introduced to be utilized in the buffer sizing procedure. Wei et al. [20] discussed the activity flexibility coefficient as a measure of the project flexibility. It can be defined as the activities associated with the resource constraint on the critical chain. In addition, the activity flexibility index can be determined depending on the importance weight of the activity. The schedule robustness can be measured by the deviation between the planned and realized activity start times during the project implementation phase. Lambrechts et al. [21] suggested the importance of the robustness measures for the buffer sizing procedure. They analyzed the application of different surrogate robustness measures to $\mathrm{CC} / \mathrm{PM}$ and project buffer sizing procedure. A surrogate robustness measure can well approximate the degree of the equivalent robustness metric.
The existing critical-chain buffer sizing approaches are mainly based on the criteria of complexity, flexibility, or their combination. Ma et al. [22] suggested an improved buffer sizing approach to the critical chain scheduling based on the flexibility concept. The proposed approach takes into account some project features including network complexity, resource tightness, and risk preference to practically size the time buffers. A critical chain-project management framework was developed by Truc et al. [23] on the basis of Max-Plus Linear algebra for handling multiple projects. The novelty was that it took into consideration the time buffer allocation decisions for both single- and multi-project cases to guarantee the due date performance. Liu et al. [24] suggested new measures for the structure entropy in order to analyze the complexity of the network in construction project schedules. In this regard, an innovative buffer sizing approach was developed that incorporated the effects of activity attributes on buffer sizing decisions. The approach proposed was verified using simulation experiments, and then it was compared with classic buffer sizing methods. Peng and Jiao [25] addressed the critical chain modeling for a multi-mode project scheduling problem. The properties of the multimode project network as well as the priority rules for activities and modes were analyzed. Peng and Huang [26] proposed an optimization approach to the generation of a buffered schedule based on the CC/PM methodology. The buffer sizing method suggested was based on the extension of the RSEM that incorporated the float time of the non-critical chains to size the feeding buffers. The resulted critical chainbased project scheduling problem was solved using a modified Differential Evolution (DE) algorithm that took into account uncertainties of the task duration. Ghamginzadeh et al. [27] addressed a multi-objective resource-constrained project scheduling problem. The objectives were the minimization of the project completion time and the time value of project costs. A multiobjective cuckoo optimization algorithm was used to find Pareto optimal solutions. Saihjpal and Singh [13] extended Cut and Paste (C\&P) method, taking into account the float time factors to decrease the project completion time and improve the project robustness. $\mathrm{Ma}$ et al. [28] improved the CC/PM methodology by integrating the buffer sizing method with multiple resource leveling techniques. The buffer allocation method proposed identifies alternative buffered schedules, taking into consideration the multiple activity execution modes. Ma et al. [29] proposed a scenariobased robust buffer sizing method that incorporates all possible scenarios of uncertain variables that may happen during the project execution phase. The $\mathrm{CC} / \mathrm{PM}$ approach proposed seeks to find a trade-off between two conflicting objectives, i.e. decreasing the 
completion time and mitigating the uncertainties of the schedule during the implementation phase.

The recent applications of $\mathrm{CC} / \mathrm{PM}$ have been considered to develop new measures to improve the performance of the generated buffered schedule. For example, a novel buffer sizing method was developed by Zhang et al. [30] aiming at improving the efficiency of the buffered schedule. They utilized project attributes including the complexity of the project network, the flexible start time of the activities, activity costs, and resource metrics to determine the size of the project buffer. Bevilacqua et al. [31] utilized goal programming methods in combination with the CC/PM methodology for RCPSP. It was assumed that the durations of the activities are random when average and standard deviations are known. The objective functions included the minimization of the project completion time and the resource leveling metric. The proposed algorithmic framework was compared with the classic PERT/CPM method, and the benefits were reported. Zhang et al. [32] developed a novel buffer sizing method based on resource tightness metrics in order to better address the relationships among activities. The Design Structure Matrix (DSM) was implemented to analyze the information flow among the activities and calculate the rework time resulting from the resource tightness metrics. Iranmanesh et al. [33] presented a buffer sizing method, taking into account the uncertainty of the duration. They proposed a Post Density Factor (PDF) that accounts for the resource constrains, location of the activity in a project network, and the risk factors. The buffer sizing method was experimentally compared with the traditional buffer sizing methods, e.g. C\&P, RSEM, APRT, and APD. Hu et al. [34] proposed an enhanced buffer control model that uses resource allocation procedures in order to minimize the total resource costs. A schedule repair approach was suggested for project re-planning when corrective actions were triggered. The test experiments demonstrated the advantages of the buffer management method over the traditional project management and control approaches. Recently, Hu et al. [35] implemented a dynamic buffer control method using the schedule risk analysis. The concept of the project control was supported by the value of the crucially index that triggers the remedial actions. Moreover, the action thresholds were dynamically adjusted in order to improve the efficiency of the buffer management approach.

Despite the wide-spread application of CC/PM to project management, this theory suffers from some deficiencies. Firstly, the theory of CC/PM focuses on the critical chain and is assumed to be constant during the project life cycle. Most of the studies on CC/PM determine the project buffer, yet the determination of the feeding buffers is always ignored. Nevertheless, in a real-world project, multiple non-critical chains have the potential of becoming critical chains, leading to delays. Also, the validity and applicability of the due date performance resulted from the buffering strategy are highly dependent on the characteristics of a project. Thus, the introduction of quantitative metrics that provides good estimates for schedule robustness is crucial for developing robust buffered project plans. The classic CC/PM approach does not properly address such issues, yet the current research tries to propose a disruption management methodology making use of efficient buffer sizing approaches. In addition, there is currently little amount of research in the field of the integrated CC/PM theory and optimization methodologies. Moreover, despite mathematical optimization models to deal with disruptions, practical solutions for this topic are still rare. Indeed, based on the discussion above, the important research gaps are highlighted as follows:

Normally, multiple initial schedules are candidates for the buffer insertion procedure. Researchers of this study attempt to develop an optimization approach to enhance the performance of the traditional $\mathrm{CC} / \mathrm{PM}$ methodology through evaluating a set of candidate schedules for the buffer allocation process:

1. Very few papers have considered the designing of the decision support system in the process of buffer sizing and buffer management subject to resource constraints;

2. Little attention has been devoted to the incorporation of optimization models together with the $\mathrm{CC} / \mathrm{PM}$ methodology.

The contributions of this research include the presentation of a novel optimization approach to the buffer allocation process as well as an effective and economic way of determining the size of the project and feeding buffers. The new iterative approach to buffer sizing explores all possible critical chains and applies the procedure to all of them. This makes it possible to obtain a better solution compared with traditional buffer sizing approaches. The model components, including a simulation tool and optimization method, are integrated with a decision support system that assists the buffer sizing decisions. The application of the decision support system is beneficial in a way that the project planner is capable of effectively measuring the stability of the critical and noncritical chains.

\section{Research methodology}

In the project management discipline, few studies are found to have been involved in the field of the integrated simulation and optimization of the project planning and control. In this research, a quantitative 
data collection and analysis approach is used, including a simulation-based optimization analysis of the schedule robustness regarding the concept of the critical chain project management. The methodology which tries to achieve the research objectives is demonstrated in Figure 1. The research methodology proposed is a multi-method approach that is comprised of a quantitative study which uses operation research techniques and a case study research.

The purpose of this study is to identify the factors affecting buffer sizes and to study the relationships among them. Using a quantitative study, the researchers of this study would know how to respond to the disruptions through academic and practical perspectives in order to get a better understanding of the problem. The purpose is to develop buffer sizing strategies for handling project delays as a contribution to the academic literature.

The techniques proposed to address the research questions include mathematical modeling as well as simulation and optimization approaches. As regards the case study of the project, the researchers of this study focused on a real-world situation aimed at gaining comprehensive understanding of the problem domain studied. The researchers of this study also introduced an algorithmic support for the traditional $\mathrm{CC} / \mathrm{PM}$ approach by presenting a multi-criteria riskbased buffer sizing model. The schedule generation approach iteratively produces potential schedules to be used as an input for the buffer sizing model.

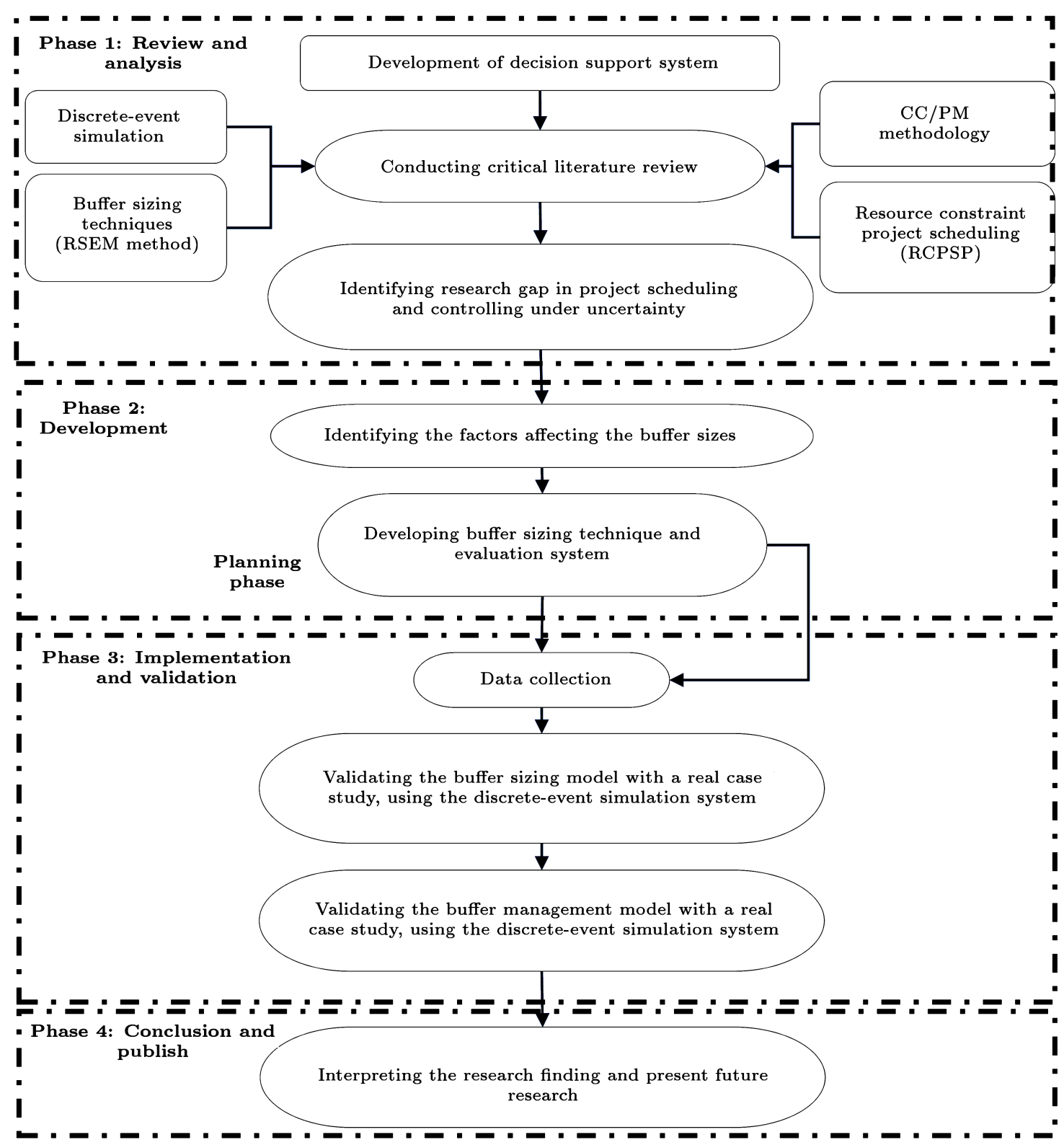

Figure 1. Stages of the research methodology proposed. 
The buffered schedules generated are then evaluated using a discrete-event simulation method and their average performance is regarded as an output. The best performing solution is then returned as the final robust schedule. The researchers of this study aimed at presenting formal theoretical representations of the relevant features and components of the problem scope in order to mitigate, analyze, and monitor delays. Furthermore, the simulation modeling methodology has also been used in the literature on the construction scheduling. The simulation technique is proposed to manipulate the disturbances and the corresponding disruptive effects. In this research, the fundamentals of the Monte-Carlo simulation methodology are also used to design some mechanisms for the effective planning and control of projects. The research methodology proposed is summarized in four phases including review and analysis, development, implementation and validation, and interpretation of the findings. The review and analysis phase (Phase 1) focuses on doing a comprehensive literature review of the state of the art of $\mathrm{CC} / \mathrm{PM}$ techniques and methodologies. The researchers of this study focus on the critical chain project management and the buffer sizing models, as well as the discrete-event simulation technique used as the validation tool.

The review and analysis stage helps to identify research gaps and shortcomings in the current practice. To answer the research questions and overcome the limitations identified in Phase 1, the major components of the DSS are identified and addressed in the development phase as follows; firstly, the methodical perspectives and their limitations are identified, representing the problem scope; secondly, the appropriate buffer sizing method is selected for improvement; thirdly, a discrete-event simulation model is developed as a validation tool. In Phase 2, the factors affecting the buffer sizes are characterized and then they are extended to multi-criteria buffer sizing model. Phase 3 involves the implementation of the developed buffer sizing method using a real case study. Finally, Phase 4 involves the presentation of the conclusions of the research. The conclusion remarks on the buffer sizing method, buffer allocation model, and buffer management method are specified. A thorough discussion of the experiments, outcomes, and lessons learnt are provided. Moreover, the conclusion provides a summary of the research findings and highlights the potential areas for further research.

\section{Buffer sizing model}

In this section, the approach proposed to model the buffer sizing process is described in detail. The multiattribute approach of this research meant to decide the buffer sizes is based on the extension of the Root Square
Error Method (RSEM). The model proposed consists of two main parts used to determine the size of the project buffer and feeding buffers. It is assumed that the inherent characteristics of the project are connected with the concepts of flexibility, robustness, complexity, and dependency.

Different factors can be considered when assigning a value to the time buffer including the level of the uncertainty of the activity duration, the project network and its degree of complexity, the float times of activities that represent the flexibility, and the resource constraints. The present study addresses the main factors affecting the buffer sizes. These factors are identified according to the literature review and realworld case study used in this study.

This study attempts to prove that the structural features of a project include the following indicators:

- Complexity indicators for the activities as well as critical and noncritical chains;

- Flexibility indicators for the critical and noncritical chains;

- Robustness indicators for the critical and noncritical chains.

\subsection{Notations}

This section is continued with the presentation of the mathematical notations. The parameters, symbols, and definitions of the attributes are summarized in Table 2.

\subsection{Network complexity and dependency criteria}

In this subsection, the complexity in the project network and its effects on the buffer sizes are analyzed. The measures proposed are within the scopes of the topological complexity of the project network and the connections between activities. Let $P_{j}$ and $S_{j}$ represent the sets of direct predecessors and successors of the $j$ th activity, respectively. The network complexity is measured using the weighted density of the precedence relationships between activities. Indeed, the number of the precedence relationships indicates that an activity can be a critical element of a project so much as to impose more delays. Here, the value of the Critical Chain Complexity $\left(C_{C C}\right)$ is defined as the maximum value for the complexity of activities on the critical chain:

$$
C_{C C}=\max _{j \in\{C C\}}\left\{\frac{\sum_{j^{\prime} \in P_{j}} \frac{\left\|s_{j^{\prime}}\right\|}{n}}{\left\|P_{j}\right\|}\right\} .
$$

Index $j^{\prime}$ was used to calculate the total number of successors of each activity that is a direct precedence of the $j$ th activity. Likewise, the Noncritical Chain Complexity index $\left(C_{N C C(i)}\right)$ is defined as the maximum 
Table 2. The symbols and notations of the buffer sizing method proposed.

\begin{tabular}{|c|c|}
\hline Symbol & Description \\
\hline$J$ & Set of activities $(j=1,2, \ldots, m+1)$ \\
\hline$Q$ & Set of resources $(q=1,2, \ldots, Q)$ \\
\hline$P_{j}$ & The set of predecessors for the $j$-th activity \\
\hline$S_{j}$ & The set of successors for the $j$-th activity \\
\hline$E S_{j}$ & Earliest start time of the $j$-th activity \\
\hline$L S_{j}$ & Latest start time of the $j$-th activity \\
\hline$E F_{j}$ & Earliest finish time of the $j$-th activity \\
\hline$T F_{j}$ & Total flotation time of the $j$-th activity \\
\hline$F F_{j}$ & Free float of the $j$-th activity \\
\hline$S F_{j}$ & Safety float of the $j$-th activity \\
\hline$d_{j}$ & The duration of the $j$-th activity \\
\hline$F_{j}$ & The finish time of task $j$ \\
\hline$r_{j q}$ & The requirement of task $j$ for resource $q$ \\
\hline$A_{q}$ & The maximum availability of resource $q$ \\
\hline$C C$ & The critical chain \\
\hline$C C_{j}$ & Criticality index for the $j$-th activity \\
\hline$C_{C C}$ & Complexity index for critical chain \\
\hline$r_{j q}$ & The resource requirement of task $j$ for resource $q$ \\
\hline$R_{q t}$ & The resource availability of task resource $q$ during time period $t$ \\
\hline$C_{N C C(i)}$ & Complexity index for the $i$-th noncritical chain \\
\hline$d_{N C C(i)}$ & The dependence index value for noncritical chain $i$ \\
\hline$M_{N C C(i)}$ & A subset of the activities on the critical chain common with non-critical activities on chain $i$ \\
\hline$\|N C C(i)\|$ & The number of activities on noncritical chain $i$ \\
\hline$D_{N C C(i)}$ & Dependence index for the $i$-th noncritical chain \\
\hline$\Delta$ & Increase or decrease rate of the buffer size according to the dependency factor \\
\hline $\begin{array}{l}M_{N C C(i)} \\
f_{C C}\end{array}$ & $\begin{array}{l}\text { The set of activities belonging to the critical chain in common with the activities on the } i \text {-th noncritical chain. } \\
\text { Flexibility value of critical chain }\end{array}$ \\
\hline$f_{N C C(i)}$ & Flexibility value of the $i$-th noncritical chain \\
\hline$F_{C C}$ & Flexibility index of critical chain \\
\hline$F_{N C C(i)}$ & Flexibility index of the $i$-th noncritical chain \\
\hline$a$ & Lower threshold value for the chain flexibility index \\
\hline$b$ & Upper threshold value for the chain flexibility index \\
\hline$f_{A}$ & The value of the normalized flexibility factor when the chain flexibility is lower than threshold value $a$ \\
\hline$f_{B}$ & The value of the normalized flexibility factor when the chain flexibility is greater than threshold value $b$ \\
\hline$a_{i}$ & Lower threshold value for the $i$-th on-critical chain flexibility index \\
\hline$b_{i}$ & Upper threshold value for the $i$-th on-critical chain flexibility index \\
\hline$\omega_{j}$ & Absolute weight for the $j$-th activity \\
\hline$\delta_{j}$ & Relative weight of the $j$-th activity on the critical chain \\
\hline$R I_{j}$ & Robustness index for the $j$-th activity \\
\hline$\beta$ & The percentage of the increase in the robustness metric \\
\hline$\eta$ & Scaling factor \\
\hline$r_{\min }$ & Minimum value of robustness index \\
\hline$R_{j}$ & The robustness criteria for the $j$-th activity \\
\hline$C V_{j}$ & Coefficients of variation \\
\hline $\operatorname{var}_{j}$ & Duration variance of the $j$-th activity \\
\hline$P B$ & The size of the project buffer \\
\hline$F B_{i}$ & The size of the $i$-th feeding buffer \\
\hline
\end{tabular}

complexity of the activities of each noncritical chain calculated as follows:

$$
C_{N C C(i)}=\max _{j \in\{N C C(i)\}}\left\{\frac{\sum_{j^{\prime} \in P_{j}} \frac{\left\|s_{j^{\prime}}\right\|}{n}}{\left\|P_{j}\right\|}\right\} \forall i .
$$

Another set of criteria related to the complexity and dependencies between activities is considered as the percentage of the overlap between a particular noncritical chain and the main critical chain. The higher this percentage is, the more indicative it is of the interdependence between the relevant noncritical chain and the critical chain.

After giving the notation, the researchers of this study define the dependence factor for the noncritical chains; the dependence factor calculates the percentage 
of the activities on the critical chain, belonging to the noncritical chain. The dependence index is always a value between zero and one $\left(0 \leq d_{N C C(i)} \leq 1\right)$ :

$$
d_{N C C(i)}=\frac{\left\|M_{N C C(i)}\right\|}{\|N C C(i)\|}
$$

To calculate the index, threshold values are required to decide on the significance degree of the dependency index on the critical chain (such as $\delta_{1}$ and $\delta_{2}$ ). If the index is greater than the bigger threshold value $\left(\delta_{2}\right)$, the buffer allocated to the critical chain will increase; otherwise, it may remain stable or decrease. Finally, the dependence index designated by symbol $D_{N C C(i)}$ is displayed in Eq. (4), and the rate of increase or decrease of the buffer size is equal to $\Delta$ :

$$
D_{N C C(i)}= \begin{cases}1-\Delta & 0 \leq d_{N C C(i)}<\delta \\ 1 & \delta_{1} \leq d_{N C C(i)} \leq \delta_{2} \\ 1+\Delta & \delta_{2} \leq d_{N C C(i)} \leq 1\end{cases}
$$

\subsection{Flexibility index}

Flexibility was broadly discussed in the field of project planning and management. The flexibility concept is closely connected with the concept of robustness. A schedule is called flexible if it can be repaired at the minimal cost. In a study carried out by Ma et al. [22], the flexibility of activities on both critical and noncritical chains is measured using the Total Flotation time (TF). However, the measure of the total float is zero for the activities on the critical path. In this study, Free Float $\left(F F_{j}\right)$ of activities on the critical chain is regarded as a measure of chain flexibility. The free float is the amount of the time that an activity can be tardy without delaying the early start of its subsequent activity. In other words, in this case, in the following activities, at the earliest possible time, $E S_{j}$ will start and the free float is calculated as follows:

$$
F F_{j}=E S_{j^{\prime} \in s_{j}}-E F_{j} .
$$

For noncritical chains, the flexibility index is calculated using the Safety Float $\left(S F_{j}\right)$ of activities. The safety float refers to the maximum time that an activity is allowed to be delayed without delaying the completion time of the project. If the activities do not necessarily delay the completion time of the project, the concept of safety float will be more suitable than the free float for the noncritical chain. Indeed, critical chain flexibility is measured as the minimum free float of the critical chain activities. If the critical chain is more flexible, fewer buffers are required:

$$
f_{C C}=\min _{j \in\{C C\}}\left\{F F_{j}\right\} .
$$

Similarly, the noncritical chain flexibility $\left(f_{N C C(i)}\right)$ is defined as the minimum safety flotation of the activities on the noncritical chain:

$$
f_{N C C(i)}=\min _{j \in\{N C C(i)\}}\left\{S F_{j}\right\} .
$$

Given the equations above, threshold values should be defined for these indicators. Therefore, the increasing or decreasing rate of the buffer time is dependent on the amount of the flexibility. Flexibility threshold values differ for critical and noncritical chains. Threshold values for the critical chain flexibility (minimum free float on the critical path activities) are considered equal to $a$ and $b$. If the critical chain flexibility is within the range $[a, b]$, the amount of flexibility will not affect the size of the buffer time. If the critical chain flexibility is less than a threshold value, ratio $f_{A}$ will be an indicator of flexibility in the buffer sizing equation. In addition, if the critical chain flexibility is greater than threshold value $b$, then ratio $f_{B}$ will be an indicator of flexibility. According to the definition, the amount of $f_{A}$ is higher than 1 and $f_{B}$ is lower than 1 . In conclusion, $F_{C C}$ denotes the normalized flexibility factor that affects the size of the time buffer:

$$
F_{C C}= \begin{cases}f_{A} & f_{C C}<a \\ 1 & a \leq f_{C C} \leq b \\ f_{B} & f_{C C}>b\end{cases}
$$

Similarly, threshold values for the noncritical chain flexibility are considered equal to $a_{i}$ and $b_{i}$ :

$$
F_{N C C(i)}= \begin{cases}f_{A}(i) & f_{N C C(i)}<a_{i} \\ 1 & a_{i} \leq f_{N C C(i)} \leq b_{i} \\ f_{B}(i) & f_{N C C(i)}>b_{i}\end{cases}
$$

\subsection{Robustness criteria}

One way of defining the degree of stability for the activities is to determine their dependence on the changes to the access to the resources and related disorders. In the literature, the Critical Index (CI) was suggested for the ratio of the average daily amount of resource requirement to the daily amount of available resources [33]. The larger the critical measure of the resources is, the higher the likelihood of delays in the project is expected to be. Previously, the impact of potential delays due to resource unavailability was measured using the Resource Tightness (RT) index. It depends on the scarcity of the resources, and therefore, the knowledge of the scarcity of the resources should be taken into account when sizing buffers. The researchers of this study proposed a modified version of RT to measure resource scarcity. In this study, the degree of robustness is calculated based on statistical measures. Robustness measures for the critical and noncritical activities will be separately described below. To develop robustness measures for activities, the researchers of this paper propose to investigate their corresponding Coefficients of Variation $\left(C V_{j}\right)$ being defined as the ratio of the standard deviation to the expected value of 
the activity duration $\left(\frac{\sigma_{j}}{\mu_{j}}\right)$. Statistically, the coefficient of variation is a measure of reliability, being indicative of the degree of uncertainty [34]. Consequently, the small value of $C V_{j}$ is desired as it indicates a slight amount of variability, so it reflects low uncertainty. In addition, the concept of resource tightness and the coefficient of variation are integrated to define the Robustness Index $\left(R I_{j}\right)$ for each activity. For the activities on the critical chain $(j \in C C)$, the robustness measure is defined as a normalized weighted coefficient of variation:

$$
R I_{j}=\frac{\delta_{j} C V_{j}}{\sum_{j^{\prime} \in\{C C\}} \delta_{j^{\prime}} C V_{j^{\prime}}} \quad j \in\{C C\} .
$$

Generally, for each activity, an absolute weight $\left(\omega_{j}\right)$ is calculated. The absolute weight indicates that for various resources required for an activity, the maximum proportion of resource requirements must be divided to the maximum level of the resource available over time. The increased weight is most likely a source of disturbances, being more desirable to be reduced:

$$
\omega_{j}=\max _{q \in Q}\left\{\frac{r_{j q}}{\min _{t \in T}\left\{R_{q t}\right\}}\right\} \quad j \in\{C C\} .
$$

Moreover, it is intended to achieve the relative weight of each activity on the critical chain $\left(\delta_{j}\right)$, so the following equation is obtained in a way that $\sum_{j \in\{C C\}} \delta_{j}=1$. The higher the relative weight for an activity is, the more it is indicative of higher dependency on the access to resources:

$$
\delta_{j}=\frac{\omega_{j}}{\sum_{j^{\prime} \in\{C C\}} \omega_{j^{\prime}}} \quad j \in\{C C\}
$$

Similarly, the robustness factors are defined for the noncritical activities. Thus, for the activities on noncritical chain, $i$, the robustness measure calculates a normalized weighted coefficient of the variation:

$$
R I_{j}=\frac{\delta_{j} C V_{j}}{\sum_{j^{\prime} \in\{N C C(i)\}} \delta_{j^{\prime}} C V_{j^{\prime}}} \quad j \in\{N C C(i)\} .
$$

The less the value of robustness index is, the less we require a buffer time. Consequently, there is a need to set the threshold values for rating the robustness index. Determining the threshold value has a significant impact on the size of the buffer. The threshold value $\left(r_{\min }\right)$ represents the minimum value of instability that leads to the increase of the buffer size. Therefore, if the indicator concerned is higher than the threshold value $\left(R I_{j}>r_{\min }\right)$, then the robustness value of each activity $\left(R_{j}\right)$ will increase by $\beta \%$ :

$$
R_{j}= \begin{cases}1+\beta & R I_{j}>r_{\min } \\ 1 & R I_{j} \leq r_{\min }\end{cases}
$$

In the following part, the final equations are provided for determining the project and feeding buffers. The project buffer time is calculated using the following equation:

$$
P B=\eta * C I_{C C} * F_{C C} * \sqrt{\sum_{j \in\{C C\}} R_{j} \cdot \operatorname{var}_{j}}
$$

where $C I_{C C}$ and $F_{C C}$ represent complexity and flexibility indices, respectively. Those two values are multiplied by the square root of the sum of the weighted variance of project activities. Similarly, for each of the noncritical chains, the feeding buffer size is determined through multiplying the weighted sum of the square root of the variances and the complexity, flexibility, risk factor, dependency factor $\left(D_{N C C(i)}\right)$, and the activity duration estimation variability $(\eta)$ :

$$
\begin{aligned}
F B_{i}= & \eta * C I_{N C C(i)} * D_{N C C(i)} * F_{N C C(i)} \\
& * \sqrt{\sum_{j \in\{N C C(i)\}} R_{j} \cdot \operatorname{var}_{j}} .
\end{aligned}
$$

\section{Algorithmic framework for $\mathrm{CC} / \mathrm{PM}$}

As already mentioned, the critical chain is determined based on both precedence dependencies and resource constraints of a single project. In the traditional CC/PM method, activities are scheduled As Late As Possible (ALAP) from the project start date. The most considerable disadvantage of planning in an ALAP policy is that in the traditional critical path method, all activities turn into critical tasks. Any increase in the duration of any activity causes the same increase in the project completion date. In this regard, time buffers can be allocated to the project network to protect the project due-date performance against stochastic variations in the activity duration. However, the benefits of CC/PM methodology are not fully exploited using a single scheduling policy (e.g., as late as possible, as soon as possible, or priority rules). Therefore, the use of proper project scheduling methods is decisive for the robustness and quality of the schedule generated, since it determines which activities are on the critical chains. Moreover, the optimization techniques assist the decision-maker in generating alternative baseline schedules, and thus improving the buffer sizing procedure through testing multiple inputs. The other important point is that the traditional CC/PM methodology suggests only the use of a single critical chain regarded to be constant during the project life cycle. Nevertheless, it can be easily confirmed that in a real-world project, more than one critical chain is nominated and the existence of different critical chains depends on the scheduling approach, objective, and scheduling policy.

The researchers of this study try to present a new iterative approach to time buffering enhanced with an optimization procedure. This approach explores all 
possible critical chains and applies the buffer sizing model to all of the candidates. In the following part, a brief summary of the optimization-based methodology of $\mathrm{CC} / \mathrm{PM}$ proposed for achieving the buffered baseline schedule is outlined (Figure 2):

1. Coming up with aggressive estimates for activity durations;

2. Constructing an initial schedule using the parallel Schedule Generation Scheme (SGS) method proposed by Kolisch and Hartmann [35];

3. Identifying the critical chains;

4. Determining appropriate buffer positions;

5. Determining appropriate buffer sizes using the proposed buffer allocation method;

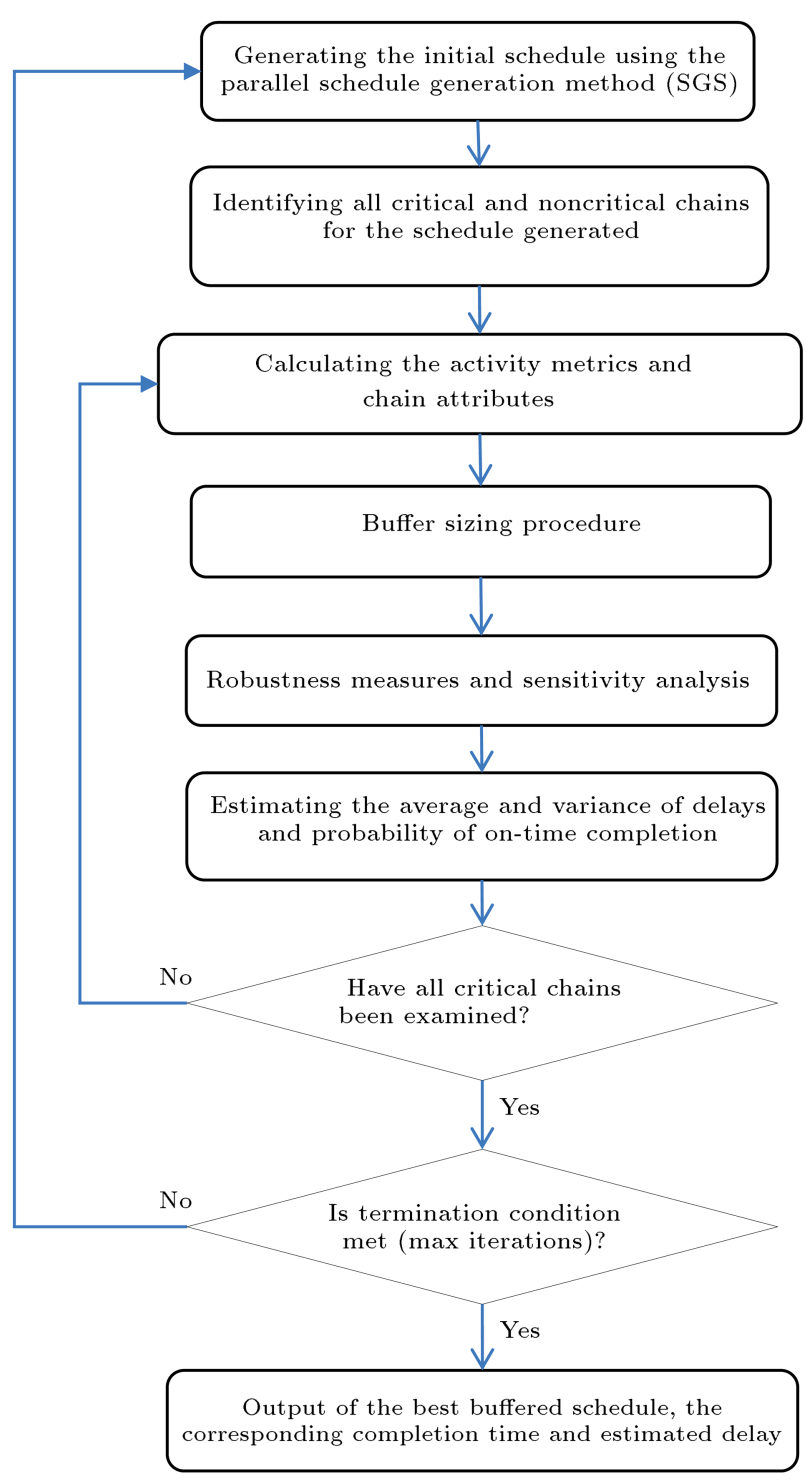

Figure 2. The flowchart of the proposed novel CC/PM methodology.
6. Validating the buffered schedule using the simulation technique;

7. Estimating the project performance in random disruption conditions;

8. The restarting of the procedure from step 2 till the termination condition is done. The termination condition is defined as the maximum number of iterations that the scheduling algorithm has implemented (the maximum number of iterations).

The procedure of identifying the critical chain is an important step in the critical chain project management. Heuristic procedures based on priority rules are the most common methods of critical chain project management; for instance, see [36]. In order to identify the critical chains, the following procedure is used. The algorithm starts with calculating the latest and earliest starting times which are then used to calculate the slack time for each activity. Activities that have zero slack time belong to the critical chain, since they are the longest resource feasible series of tasks in the network. Accordingly, assume that for each task, $j$, the early starting times $\left(E S_{j}\right)$ are given. Let $e_{t}$ be a variable for event times. The researchers of this study define the following mutually exclusive sets. The scheduled set is represented by $C_{t}=\left\{j \in J \mid L S_{j} \geq e_{t}\right\}$. It consists of activities that have been scheduled to start at or after time $e_{t}$. The active set consists of activities started prior to time $e_{t}$ and still active at time $e_{t}$ expressed by set $\Delta_{t}=\left\{j \in J \mid L S_{j}<e_{t} \leq L S_{j}+d_{j}\right\}$. The remaining capacity of resource $q$ at time $t$ is $\bar{R}_{q}(t)$; thus, at time $e_{t}$, it is $\bar{R}_{q}\left(e_{t}\right)=A_{q}-\sum_{j \in \Delta_{t}^{r_{j q}}}$. Accordingly, eligible set $E$ consists of the tasks which are either inactive or not scheduled at time $e_{t}$, but their successors had been scheduled after time $e_{t}$, The resource feasibility is tested not only at time $e_{t}$, but over the durations. Consequently, it is feasible to delay all the tasks in set $E$ to be completed at time $e_{t}$ :

$$
\begin{aligned}
E_{t}= & \left\{j \in J \backslash\left(E_{t} \cup \Delta_{t}\right) \mid\left(S_{j} \subseteq C_{t}\right)\right. \text { and } \\
& \left.\left(\sum_{j} r_{j q} \leq \bar{R}_{q}\left(e_{t}\right), q \in Q \text { and }\left(\bar{R}_{q}(t) \geq 0 \forall t\right)\right)\right\} .
\end{aligned}
$$

After the above notations and definitions, the algorithm described below is used to identify the critical chain activities:

Initialization step: $L S_{j}=E S_{j} \forall j, \Delta_{0}=\{m+1\}$, $C_{0}=\phi, t=1$

Step 1: While $\left|\Delta_{t-1} \cup C_{t-1}\right| \leq m+1$

Step $1.1 e_{t}=\max _{j \in \Delta_{t-1}}\left\{L S_{j}\right\}$

Update $C_{t}, \Delta_{t}, \bar{R}_{q}\left(e_{t}\right), E_{t}$

Step 1.2 While $E_{t} \neq \phi$ do

Select one activity that satisfies $i \in E_{t}$ Let $L F_{j}=e_{t}$ 
Step $1.3 t=t+1$

$$
\begin{aligned}
& \text { Let } L S_{j}=e_{t}-d_{j} \\
& \text { Let } \Delta_{t}=\Delta_{t} \cup\{j\} \\
& \text { Let } E_{t}=E_{t} \backslash\{j\}
\end{aligned}
$$

Step 2: For $j=1$ to $m+1$

$$
\text { If }\left(L S_{j}=E S_{j}\right) \text { then } j \in C C
$$

In order to generate multiple candidate schedules for buffer allocation, we implement a solution generation procedure which is a minor modification of the parallel Schedule Generation Scheme (SGS) of Hartmann and Kolisch [37]. The method is based on a forward mechanism to schedule eligible tasks starting at time $t=0$. The modification is met by the definition of critical tasks based on their priorities. The tasks along the critical chain are given priority and scheduled as soon as possible in the order in which they appear on the critical chain. However, the tasks not belonging to the critical chain, but eligible, are scheduled according to the baseline schedule. The complete set includes the activities completed at or before time $e_{t}$ which is represented by $C_{t}=\left\{j \in J \mid F_{j} \leq e_{t}\right\}$. The activities in the active set have been started at or before time $e_{t}$, but not yet completed. Therefore, we have: $\Delta_{t}=\{j \in$ $\left.J \mid F_{j}-d_{j} \leq e_{t}<F_{j}\right\}$. With the above notation, we defined the prioritized eligible set as follows:

$$
\begin{aligned}
E_{t}=E_{t}^{+}= & \left\{j \in J \backslash\left(E_{t} \cup \Delta_{t}\right) \mid\left(P_{j} \subseteq C_{t}\right)\right. \text { and } \\
& \left(\sum_{j} r_{j q} \leq \bar{R}_{q}\left(e_{t}\right), q \in Q\right. \\
& \text { and }(j \in C C))\} .
\end{aligned}
$$

Un-prioritized eligible set is also formed as follows:

$$
\begin{aligned}
E_{t}^{+}= & \left\{j \in J \backslash\left(E_{t} \cup \Delta_{t}\right) \mid\left(P_{j} \subseteq C_{t}\right)\right. \text { and } \\
& \left.\left(\sum_{j} r_{j q} \leq \bar{R}_{q}\left(e_{t}\right), q \in Q \text { and }(j \notin C C)\right)\right\} .
\end{aligned}
$$

The following algorithm gives priority to activities in the critical chain. Therefore, the activities meeting the precedence feasibility of the critical chain are the first ones to be scheduled during the resource allocation procedure.

Initialization step: $\Delta_{0}=\{0\}, C_{0}=\phi, F_{0}=0, g=1$ Step 1: While $\left|\Delta_{t-1} \cup C_{t-1}\right| \leq m+1$ do

Step $1.1 e_{t}=\min _{j \in \Delta_{t-1}}\left\{F_{j}\right\}$ Update the value of $C_{t}, \Delta_{t}, \bar{R}_{q}\left(e_{t}\right)$, $E_{t}^{+}, E_{t}^{-}$

Step 1.2 While $E_{t}^{+} \neq \phi$ do Select one that satisfies $j \in E_{t}^{+}$

$$
\begin{aligned}
& F_{j}=e_{t}+d_{j} \\
& \Delta_{t}=\Delta_{t} \cup\{j\} \\
& E_{t}^{+}=E_{t}^{+} \backslash\{j\} \\
& \text { Update } \bar{R}_{q}\left(e_{t}\right), E_{t}^{-}
\end{aligned}
$$

Step 1.3 While $E_{t}^{-} \neq \phi$ do

Select one that satisfies $j \in E_{t}^{-}$

$$
\begin{aligned}
& F_{j}=e_{t}+d_{j} \\
& \Delta_{t}=\Delta_{t} \cup\{j\} \\
& E_{t}^{-}=E_{t}^{-} \backslash\{j\}
\end{aligned}
$$$$
\text { Step } 1.4 t=t+1
$$

Terminate: Provide the actual project completion time as $F_{m+1}$.

\section{Illustrative example}

In this section, the steps motioned above are applied to an illustrative example of the project network presented in Figure 3. All empirical experiments are executed on an Intel(R) Core2 Due Personal Computer (PC), with the $3.3 \mathrm{GHz} \mathrm{CPU}$ and an $8 \mathrm{~GB}$ memory on a Windows 8 platform. The scheduling algorithm was programmed using MATLAB R2015b software. In the example adopted from [38], three renewable resource types are utilized, namely $\mathrm{A}, \mathrm{B}$, and $\mathrm{C}$, with the maximum availability of 3,1 , and 2 units over the planning time span, respectively.

The activity durations and the resource requirements for three renewable resource types are represented in Table 3 . The start and end activities are dummies, representing the milestones of the project. The duration of each activity can be modeled as a stochastic variable with a given probability density function where the variance differs for the activities. The present CC/PM starts by generating a resourcefeasible schedule that can be attained by a "SGS" method.

\subsection{Experimental result}

The complexity of the relationship between project activities in numerical examples is summarized in Table 4. This table includes the percentage of activities after the critical need, from the perspective of the relationship between the activities, calculated for each activity. The activities of the project, i.e. activities 1 and 2, have the largest values for their complexity indices. As expected, the complexity index for the initial activities and the project is rising as we move closer to the end of the project, and the complexity of the relationship between the activities is relatively reduced.

The complexity index changes as a function of the number of precedence relationships, so do the activities as outlined in Figure 4. As expected, the initial project activities account for a greater percentage of the structural complexity of the activities. However, due to the 


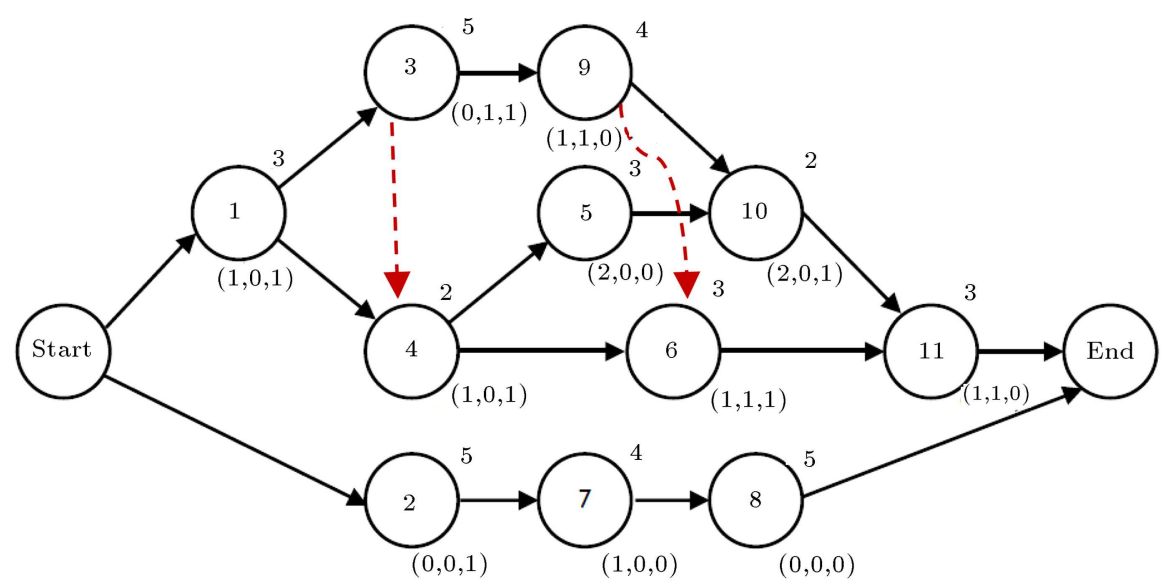

Figure 3. The precedence and resource-dependent relationship between activities in the example adopted from [38].

Table 3. The information of the project activities.

\begin{tabular}{ccccccc}
\hline $\begin{array}{c}\text { Activity } \\
\text { number } \\
(\boldsymbol{j})\end{array}$ & $\begin{array}{c}\text { Average } \\
\text { duration } \\
\left(\boldsymbol{\mu}_{\boldsymbol{j}}\right)\end{array}$ & $\begin{array}{c}\text { Variance of } \\
\text { duration } \\
\left(\mathbf{v a r}_{\boldsymbol{j}}\right)\end{array}$ & $\begin{array}{c}\text { Coefficient of } \\
\text { variation } \\
\left(\boldsymbol{C} \boldsymbol{V}_{\boldsymbol{j}}\right)\end{array}$ & $\begin{array}{c}\text { Resource } \\
\text { usage } \\
(\mathbf{A})\end{array}$ & $\begin{array}{c}\text { Resource } \\
\text { usage } \\
(\mathbf{B})\end{array}$ & $\begin{array}{c}\text { Resource } \\
\text { usage } \\
(\mathbf{C})\end{array}$ \\
\hline 0 & 0 & 0 & 0.4 & 0 & 0 & 0 \\
1 & 3 & 1.2 & 0.4 & 1 & 0 & 1 \\
2 & 5 & 2 & 0.4 & 0 & 0 & 1 \\
3 & 5 & 2 & 0.4 & 0 & 1 & 1 \\
4 & 2 & 0.8 & 0.4 & 1 & 0 & 1 \\
5 & 3 & 1.2 & 0.4 & 2 & 0 & 0 \\
6 & 3 & 1.2 & 0.4 & 1 & 1 & 1 \\
7 & 4 & 1.6 & 0.4 & 1 & 0 & 0 \\
8 & 5 & 2 & 0.4 & 0 & 0 & 0 \\
9 & 4 & 1.6 & 0.4 & 1 & 1 & 0 \\
10 & 2 & 0.8 & 0.4 & 2 & 0 & 1 \\
11 & 3 & 1.2 & 0.4 & 1 & 1 & 0 \\
12 & 0 & 0 & 0.4 & 0 & 0 & 0 \\
\hline
\end{tabular}

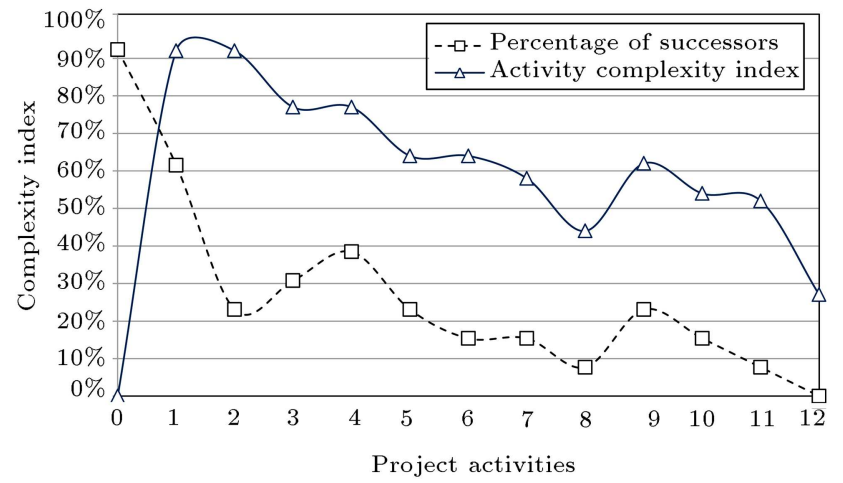

Figure 4. The complexity index calculated for activities.

complexity of the activities of the function definition required, this process is associated with fluctuations.

Flexibility and safety analyses and the associated calculation of free float times for project activities are given in numerical examples in Table 5. Critical Chain Flexibility $\left(F l e x_{C C}\right)$ is equal to the minimum free float on the critical path activities. If the minimum free float of activities on the critical chain exceeds the threshold value, we will require less safety time. The flexibility index for the critical chain is calculated as follows. Threshold values for parameters $a=0.5$ and $b=2.5$ are taken into account. As a result, if the flexibility index of the critical chain is within the range $[a, b]$, the flexibility will not change the size of the safety time. If some flexibility indices of the critical chain are fewer than the threshold value, the coefficient of the flexibility, in relation to the size of the safety stock index, shall be equal to $f_{A}=1.1(10 \%$ increase). Likewise, if the flexibility of the critical chain is more than threshold value $b=2.5$, the coefficient 
Table 4. The results of complexity analysis.

\begin{tabular}{ccccc}
\hline $\begin{array}{c}\text { Activity } \\
\text { number }\end{array}$ & $\boldsymbol{S}_{\boldsymbol{j}}$ & $\boldsymbol{C}_{\boldsymbol{j}}$ & $\left\|\boldsymbol{P}_{\boldsymbol{j}}\right\|$ & $\begin{array}{c}\text { Activity } \\
\text { complexity }_{\boldsymbol{j}}\end{array}$ \\
\hline 0 & 12 & $92.31 \%$ & 0 & 0 \\
1 & 8 & $61.54 \%$ & 1 & 0.92 \\
2 & 3 & $23.08 \%$ & 1 & 0.92 \\
3 & 4 & $30.77 \%$ & 2 & 0.77 \\
4 & 5 & $38.46 \%$ & 2 & 0.77 \\
5 & 3 & $23.08 \%$ & 3 & 0.64 \\
6 & 2 & $15.38 \%$ & 3 & 0.64 \\
7 & 2 & $15.38 \%$ & 2 & 0.58 \\
8 & 1 & $7.69 \%$ & 3 & 0.44 \\
9 & 3 & $23.08 \%$ & 3 & 0.62 \\
10 & 2 & $15.38 \%$ & 4 & 0.54 \\
11 & 1 & $7.69 \%$ & 4 & 0.52 \\
12 & 0 & $0.00 \%$ & 12 & 0.27 \\
\hline
\end{tabular}

Table 5. Analysis of flexibility for project activities in the numerical example.

\begin{tabular}{ccccc}
\hline Activity number & $\boldsymbol{E} \boldsymbol{S}_{\boldsymbol{j}}$ & $\boldsymbol{E}_{\boldsymbol{j}}$ & $\boldsymbol{F}_{\boldsymbol{j}}$ & $\boldsymbol{S}_{\boldsymbol{j}}$ \\
\hline 0 & 0 & 0 & 0 & 0 \\
1 & 0 & 3 & 0 & 0 \\
2 & 0 & 5 & 4 & 3 \\
3 & 3 & 8 & 0 & 0 \\
4 & 8 & 10 & 3 & 1 \\
5 & 10 & 13 & 1 & 1 \\
6 & 12 & 15 & 0 & 0 \\
7 & 5 & 9 & 4 & 3 \\
8 & 13 & 18 & 4 & 4 \\
9 & 8 & 12 & 0 & 0 \\
10 & 12 & 14 & 2 & 2 \\
11 & 15 & 18 & 0 & 0 \\
12 & 18 & 18 & 1 & 1 \\
\hline
\end{tabular}

Table 6. Calculating the flexibility of non-critical chain.

\begin{tabular}{cccc}
\hline Non-critical chains & $\boldsymbol{F}_{\mathbf{1}}$ & $\boldsymbol{F}_{\mathbf{2}}$ & $\boldsymbol{F}_{\mathbf{3}}$ \\
\hline Flex $_{N C C(i)}$ & 1 & 1 & 3 \\
$F_{N C C(i)}$ & 1 & 1 & 0.9 \\
\hline
\end{tabular}

of the flexibility index will be equal to $f_{B}=0.90$ (10\% decrease). According to Eq. (8), the coefficient of the flexibility index for the critical chain is obtained $(F C C=1)$. For noncritical chains, the values are also summarized in Table 6.

For the stability analysis, the variation coefficient of activities was used. The absolute weight factor, being closely related to the use of the resource available to the maximum value, was obtained. The relative weight normalized for each activity on the critical
Table 7. Robustness analysis for the numerical example.

\begin{tabular}{ccccc}
\hline Activity number & $\boldsymbol{\omega}_{\boldsymbol{j}}$ & $\boldsymbol{\delta}_{\boldsymbol{j}}$ & $\boldsymbol{C}_{\boldsymbol{j}}$ & $\boldsymbol{R I}_{\boldsymbol{j}}$ \\
\hline 1 & 0.5 & 0.11 & 0.4 & 0.11 \\
3 & 1 & 0.22 & 0.4 & 0.22 \\
6 & 1 & 0.22 & 0.4 & 0.22 \\
9 & 1 & 0.22 & 0.4 & 0.22 \\
11 & 1 & 0.22 & 0.4 & 0.22 \\
\hline
\end{tabular}

Table 8. Stability analysis for the activities of non-critical chain.

\begin{tabular}{ccccc}
\hline $\begin{array}{c}\text { Non-critical } \\
\text { chain }\end{array}$ & $\begin{array}{c}\text { Activity } \\
\text { number }\end{array}$ & $\boldsymbol{\omega}_{\boldsymbol{j}}$ & $\boldsymbol{\delta}_{\boldsymbol{j}}$ & $\boldsymbol{R}_{\boldsymbol{j}}$ \\
\hline & 1 & 0.5 & 0.17 & 0.17 \\
1 & 4 & 0.5 & 0.17 & 0.17 \\
& 6 & 1 & 0.33 & 0.33 \\
& 11 & 1 & 0.33 & 0.33 \\
& 1 & 0.5 & 0.15 & 0.15 \\
& 4 & 0.5 & 0.15 & 0.15 \\
2 & 5 & 0.67 & 0.2 & 0.2 \\
& 10 & 0.67 & 0.2 & 0.2 \\
& 11 & 1 & 0.3 & 0.3 \\
& & & & \\
3 & 2 & 0.5 & 0.6 & 0.6 \\
& 7 & 0.33 & 0.4 & 0.4 \\
& 8 & 0 & 0 & 0 \\
\hline
\end{tabular}

chain $\left(\delta_{j}\right)$ was calculated. The results of the stability measurement of the critical chain project activities in the numerical example are given in Table 7 . The variation coefficient in this example equals 0.4 . In this particular case, the standard deviation of all activities is the same, with the relative stability for each activity $\left(R I_{j}\right)$ being equal to the relative weight factor $\left(\delta_{j}\right)$. Similarly, the results of the stability analysis for the activities of non-critical chain, utilizing numerical examples, are summarized in Table 8.

\subsection{Buffer sizing}

In this section, the project and feeding buffers are determined, and the respective values are allocated. Before presenting the empirical results, the benchmark methods are discussed. The buffer size is typically determined in the following two kinds of methods: C\&P and RSEM methods. The C\&P method utilizes the $50 \%$ and $90 \%$ estimations of the activity duration. Accordingly, the buffer size equals half of the sum of the difference between $90 \%$ of the completion probability of the activity duration estimated $\left(t_{90 \%}(j)\right)$ and $50 \%$ of the completion probability of the activity duration estimated $\left(t_{50 \%}(j)\right)$ as follows:

$$
\text { Buffer size }=\frac{1}{2}\left(\sum_{j \in J} t_{90 \%}(j)-t_{50 \%}(j)\right) \text {. }
$$

The RSEM method is based on the independent assumption of the project activities. The RSEM uses 
the statistical information of the project activities to determine the size of the buffer as follows:

$$
\text { Buffer size }=\sqrt{\left(\sum_{j \in J} t_{90 \%}(j)-t_{50 \%}(j)\right)} .
$$

According to the methodology proposed, the buffer time allocated to the critical chain is calculated as follows:

$$
\begin{aligned}
P B & =\eta * C R * C I_{C C} * F_{C C} * \sqrt{\sum_{j \in\{C C\}} R_{j} \cdot \sigma_{j}^{2}} \\
& =2 * 1 * 1.1 * 1 \\
& * \sqrt{1.2^{2}+2^{2}+1.6^{2}+1.05 * 1.2^{2}+1.05 * 1.2^{2}} \\
& =7.3 .
\end{aligned}
$$

For each non-critical chain project, the following formula is used to calculate the buffer sizes; the corresponding results are also summarized in Table 9 :

$$
\begin{aligned}
F B_{1}= & \eta \cdot C R * C I_{N C C(i)} * D_{N C C(i)} * F_{N C C(i)} \\
& * \sqrt{\sum_{j \in\{N C C(i)\}} R_{j} \cdot \sigma_{j}^{2}}=2 * 1 * 1.1 * 1.1 * 1 \\
& * \sqrt{0.8^{2}}=1.9, \\
F B_{2}= & \eta \cdot C R * C I_{N C C(i)} * D_{N C C(i)} * F_{N C C(i)} \\
& * \sqrt{\sum_{j \in\{N C C(i)\}} R_{j} \cdot \sigma_{j}^{2}}=2 * 1 * 1.1 * 1 * 1 \\
& * \sqrt{2^{2}+1.6^{2}+2^{2}}=7.15, \\
F B_{3}= & * C R * C I_{N C C(i)} * D_{N C C(i)} * F_{N C C(i)} \\
& * \sqrt{\sum_{j \in\{N C C(i)\}} R_{j} \cdot \sigma_{j}^{2}}=2 * 1 * 1.1 * 0.9 * 0.9 \\
& * \sqrt{1.05 * 0.8^{2}+1.05 * 1.2^{2}+0.8^{2}}=3 .
\end{aligned}
$$

According to the results presented in Table 9, the project buffer size increases by $10.6 \%$; also, the feeding buffers for the non-critical chain increase by $18.75 \%$ and $10.00 \%$ for the first and second noncritical chains, respectively, using the method proposed. As it can be seen, the size of the feeding buffers increased except for the one (Feeding buffer 3 ) that has been reduced by approximately $9 \%$. In addition, the buffer time obtained using the method proposed is by $7.5 \%$ greater than the value obtained using the RSEM method.

\subsection{Validation}

The validation process for the buffered schedule is carried out using a discrete-event simulation model (Monte-Carlo simulation method). In the simulation model, random disturbances for the duration of project activities are created, and the completion time is calculated. The Monte-Carlo method is commonly used for risk analysis and project planning issues [39]. The researchers of this study used this method for the real-time distributed simulation and analysis of disruption consequences as well as deviation from the initial program to evaluate the initial schedule [40]. If the actual completion date of the project after simulation exceeded the planned completion date, the project delay would occur and calculations would be recorded. Thus, the researchers of this study can calculate the risk of project delays and obtain the mean and variance. The disruption is defined in a way that $\alpha$ percent of the project activities is selected, and $\beta$ is added to their times. This definition has been considered in various studies [41].

Because of the stochastic nature of the simulation models, a single outcome is not representative. Consequently, a number of observations can be mandatory in order to obtain a reliable consequence with desirable levels of accuracy. The reliability of the results is represented by the Confidence Interval (CI) indicating the probability that the output variable is within the range specified. During the simulation process, an observation, like $w_{i}$, is carried out after each observation period $i$. Each statistic is estimated based on the raw data, $w_{1}, w_{2}, \ldots, w_{n}$, where $n$ is the number of observation periods (number of replications). The lower and upper bounds of the confidence interval are obtained from Eq. (26). Values $t_{n-1,1-\frac{1}{2} \alpha}$ and $\mu_{1-\frac{1}{2} \alpha}$ are obtained from a table of $t$-values, where $\alpha=1-$ Reliability:

Table 9. The results of the buffer sizes obtained by the proposed method and the benchmark approach.

\begin{tabular}{cccccc}
\hline $\begin{array}{c}\text { Buffer sizing } \\
\text { approach }\end{array}$ & $\begin{array}{c}\text { Project } \\
\text { buffer }\end{array}$ & $\begin{array}{c}\text { Feeding } \\
\text { buffer 1 }\end{array}$ & $\begin{array}{c}\text { Feeding } \\
\text { buffer 2 }\end{array}$ & $\begin{array}{c}\text { Feeding } \\
\text { buffer 3 }\end{array}$ & $\begin{array}{c}\text { Total buffer } \\
\text { (days) }\end{array}$ \\
\hline RSEM & 6.6 & 1.6 & 6.5 & 3.3 & 18 \\
Method proposed & 7.3 & 1.9 & 7.15 & 3 & 19.35 \\
Change percentage & $10.61 \%$ & $18.75 \%$ & $10.00 \%$ & $-9.09 \%$ & $7.50 \%$ \\
\hline
\end{tabular}


Confidence interval

$$
= \begin{cases}\bar{w} \pm t_{n-1,1-\frac{1}{2} \alpha^{\prime}} \frac{S}{\sqrt{n}} & n \leq 30 \\ \bar{w} \pm \mu_{1-\frac{1}{2} \alpha^{\prime}} \frac{S}{\sqrt{n}} & n>30\end{cases}
$$

The discrete event simulation model was implemented for $n=100$ replications. The result of the schedule stability analysis, using a simulation approach, is presented in Table 10. According to different random disturbances, the expected delay, its variance, and the probability of on-time project completion were reported. The result exhibits the performance of the identified methods for determining the size of the buffer, where the proposed method is compared with them. The results show the superiority of the proposed method for determining the buffer size compared with RSEM with about $5.2 \%$ reduction of the possibility of the delay as well as $30 \%$ reduction in the average time delay. The delay variance of the project completion time for the schedule obtained from the proposed method decreased by about $60 \%$. The results show the superiority of the proposed method over RSEM. The method proposed here is also compared with C\&P. It should be noted that the use of C\&P leads to incurring high costs because the buffer sizes are overestimated and the activities should be programmed considering $50 \%$ of the average estimate. In fact, although the results of $\mathrm{C} \& \mathrm{P}$ reach a high degree of probability, the project is completed on time. However, this time with the buffer allocated (about two times the buffer obtained from the method proposed) has been obtained.

According to the index of the project, the project and feeding buffers values can be selected for different scenarios to obtain the critical chain buffers. When critical chain project " $1-3-4-5-8$ " is selected, the buffer sizes are calculated as follows:

$$
\begin{aligned}
& P B_{1-3-4-5-8}=\eta * C R * C I_{C C} * F_{C C} \\
& * \sqrt{\sum_{j \in\{C C\}} R_{j} \cdot \sigma_{j}^{2}}=2 * 1 * 1.1 * 1 \\
& * \sqrt{1.2^{2}+1.05 * 2^{2}+0.8^{2}+1.05 * 1.2^{2}+2^{2}} \\
& =7.55 .
\end{aligned}
$$

On the other hand, with the critical chain selected as "1-3-4-5-10-11", the project buffer time is calculated as follows:

$$
\begin{aligned}
& P B_{1-3-4-5-10-11}=\eta * C R * C I_{C C} * F_{C C} \\
& * \sqrt{\sum_{j \in\{C C\}} R_{j} \cdot \sigma_{j}^{2}}=2 * 1 * 1.1 * 1 \\
& * \sqrt{1.2^{2}+1.05 * 2^{2}+0.8^{2}+1.05 * 1.2^{2}+0.8^{2}+1.05 * 1.2^{2}} \\
& =6.93 .
\end{aligned}
$$

In the critical chain "1-3-4-5-8", for each non-critical chain project, the size of feeding buffer is calculated using the following equation:

$$
\begin{aligned}
F B_{1}= & \eta C R * C I_{N C C(i)} * D_{N C C(i)} * F_{N C C(i)} \\
& * \sqrt{\sum_{j \in\{N C C(i)\}} R_{j} \cdot \sigma_{j}^{2}}=2 * 1 * 1.1 * 0.9 * 1 \\
& * \sqrt{0.8^{2}}=1.58, \\
F B_{2}= & \eta C R * C I_{N C C(i)} * D_{N C C(i)} * F_{N C C(i)} \\
& * \sqrt{\sum_{j \in\{N C C(i)\}} R_{j} \cdot \sigma_{j}^{2}}=2 * 1 * 1.1 * 0.9 * 1 \\
& * \sqrt{2^{2}+1.6^{2}+2^{2}}=6.43, \\
F B_{3}= & * C R * C I_{N C C(i)} * D_{N C C(i)} * F_{N C C(i)} \\
& * \sqrt{\sum_{j \in\{N C C(i)\}} R_{j} \cdot \sigma_{j}^{2}}=2 * 1 * 1.1 * 0.9 * 0.9 \\
& * \sqrt{1.05 * 0.8^{2}+1.05 * 1.2^{2}+0.8^{2}}=3 .
\end{aligned}
$$

In critical chain "1-3-4-5-10-11", as seen in the calculation above, the size of the feeding buffers is marked. The results for all three possible options for the critical chain and the corresponding buffer times are summarized in Table 11. As seen, the change in the

Table 10. Results of buffer sizing approach to the numerical example ( $\alpha=0.05)$.

\begin{tabular}{ccccccc}
\hline $\begin{array}{c}\text { Buffer sizing } \\
\text { approach }\end{array}$ & $\begin{array}{c}\text { Probability of } \\
\text { on-time project } \\
\text { completion time }\end{array}$ & $\begin{array}{c}\text { Average } \\
\text { delay } \\
\text { (days) }\end{array}$ & $\begin{array}{c}\text { delay } \\
\text { (days) }\end{array}$ & $\begin{array}{c}\text { on project } \\
\text { delay }\end{array}$ & $\begin{array}{c}\text { on project } \\
\text { delay }\end{array}$ & $\begin{array}{c}\text { Total } \\
\text { buffer }\end{array}$ \\
\hline RSEM & $91.10 \%$ & 1.34 & 3.81 & 0.169 & 2.511 & 6.6 \\
C\&P & $98.50 \%$ & 0.87 & 0.62 & 0.398 & 1.342 & 14 \\
Robust buffer sizing method & $95.70 \%$ & 0.93 & 1.57 & 0.178 & 1.682 & 7.3 \\
\hline
\end{tabular}


Table 11. The results of buffer allocation.

\begin{tabular}{cccccc}
\hline $\begin{array}{c}\text { Critical chain } \\
\text { candidates }\end{array}$ & $\begin{array}{c}\text { Project } \\
\text { buffer }\end{array}$ & $\begin{array}{c}\text { Feeding } \\
\text { buffer 1 }\end{array}$ & $\begin{array}{c}\text { Feeding } \\
\text { buffer 2 }\end{array}$ & $\begin{array}{c}\text { Feeding } \\
\text { buffer 3 }\end{array}$ & $\begin{array}{c}\text { Total } \\
\text { buffer } \\
\text { (days) }\end{array}$ \\
\hline "1-3-4-5-8" & 7.55 & 1.58 & 6.43 & 3 & 18.56 \\
"1-3-4-5-10-11" & 6.93 & 1.58 & 6.43 & 3 & 17.94 \\
"1-3-9-6-11" & 7.3 & 1.9 & 7.15 & 3 & 19.35 \\
\hline
\end{tabular}

Table 12. Results of buffer sizing approach on the illustrative example.

\begin{tabular}{ccccc}
\hline $\begin{array}{c}\text { Critical chain } \\
\text { candidates }\end{array}$ & $\begin{array}{c}\text { Probability of } \\
\text { on-time project } \\
\text { completion time }\end{array}$ & $\begin{array}{c}\text { Average } \\
\text { delay } \\
\text { (days) }\end{array}$ & $\begin{array}{c}\text { Variance of } \\
\text { delay } \\
\text { (days) }\end{array}$ & $\begin{array}{c}\text { Total } \\
\text { buffer }\end{array}$ \\
\hline "1-3-4-5-8" & $96.40 \%$ & 0.9 & 1.5 & 7.55 \\
"1-3-4-5-10-11" & $94.90 \%$ & 1.08 & 1.72 & 6.93 \\
"1-3-9-6-11" & $95.70 \%$ & 0.93 & 1.57 & 7.3 \\
\hline
\end{tabular}

buffer size can be negligible by changing the critical chain.

After evaluating and comparing the plans based on scheduler choices, it was concluded that if chain "1-3-4-5-10-11" is selected, the probability of on-time project completion time will be lower than other states (Table 12). In addition, critical chain "1-3-4-5-8" had a slight increase of approximately $0.73 \%$ for reliability as against the initial state (less than 1\%).

\section{Conclusion}

Projects are subject to various sources of uncertainties that often negatively affect activity durations and costs. Therefore, it is of crucial importance to develop a decision support system to predict the potential risks and efficiently manage disruptions during planning and execution phases.

To further advance research in the field of schedule management and to improve the effectiveness of the framework proposed, future research should focus more on clarifying the uncertainty metrics to better assess the severity of the effects of various uncertainties; it must also focus on increasing the number of activity modes to better reflect how activities are performed in practice and to enrich the options that project teams have at their disposal for adjusting resource profiles.

Moreover, further research should examine the dynamic updating of buffer sizes during the project implementation phase in such a manner that project teams can be prepared when risks change due to the modification of project environments and ongoing work progress. In addition, future research can be performed to examine the potentials of multi-objective optimization, which will consider not only the project duration, but also other objectives such as cost control and logistics management, and to realize methodical and comprehensive project management criteria.

The constraints that limit the implementation of the research in this field are listed below:

1. The availability of the real disruption data and the relevant consequences is of high concern;

2. In addition, the costs of information collection during the dynamic nature of the projects limit the application of the project control procedure;

3. There is also a challenge of the demonstration of the application of the proposed algorithm in the multi-project environment;

4. Finally, the decision support system must gather proper information on the deviation as well as overruns for real-time cases.

In this research, simulation modeling techniques were used to give credit to the results of buffer sizing method. The discrete-event simulation technique has several major drawbacks such as its limited capability of capturing the strategic perspectives in the context of project management and its incapability of addressing the cause-effect relationships that exist between project elements. Due to limitations of the discrete-event simulation methods in the problem solving domain, a hybrid simulation-optimization platform is required.

\section{References}

1. Huang, G.Q., Lau, J.S., Mak, K.L. and Liang, L. "Distributed supply-chain project rescheduling: Part I-impacts of information-sharing strategies", International Journal of Production Research, 43(24), pp. 5107-5129 (2005). 
2. Kao, H.-P., Hsieh, B. and Yeh, Y. "A petri-net based approach for scheduling and rescheduling resourceconstrained multiple projects", Journal of the Chinese Institute of Industrial Engineers, 23(6), pp. 468-477 (2006).

3. Kuster, J., Jannach, D. and Friedrich, G. "Applying local rescheduling in response to schedule disruptions", Annals of Operations Research, 180(1), pp. 265-282 (2010).

4. Larsen, R. and Pranzo, M. "A framework for dynamic rescheduling problems", Journal of Scheduling, 139, pp. 230-244 (2012).

5. Liu, S.-S. and Shih, K.-C. "Construction rescheduling based on a manufacturing rescheduling framework", Automation in Construction, 18(6), pp. 715723 (2009).

6. Wynn, D.C. and Clarkson, P.J. "Design project planning, monitoring and re-planning through process simulation", in DS 58-1: Proceedings of ICED 09, the 17th International Conference on Engineering Design, 1, Design Processes, Palo Alto, CA, USA (2009).

7. Kerzner, H.R., Project Management: A Systems Approach to Planning, Scheduling, and Controlling, John Wiley \& Sons (2013).

8. Zhu, G., Bard, J.F. and Yu, G. "Disruption management for resource-constrained project scheduling", Journal of the Operational Res earch Society, 56(4), pp. 365-381 (2005).

9. Yu, G. and Qi, X., Disruption Management: Framework, Models and Applications, World Scientific Publishing Company Incorporated (2004).

10. Herroelen, W. and Leus, R. "The construction of stable project baseline schedules", European Journal of Operational Research, 156(3), pp. 550-565 (2004).

11. Lambrechts, O., Demeulemeester, E., and Herroelen, W. "Time slack-based techniques for robust project scheduling subject to resource uncertainty", Annals of Operations Research, 186(1), pp. 443-464 (2011).

12. Russell, M.M., Hsiang, S.M., Liu, M. and Wambeke, B. "Causes of time buffer and duration variation in construction project tasks: Comparison of perception to reality", Journal of Construction Engineering and Management, 140(6), pp. 04014016-1-04014016-12 (2014).

13. Saihjpal, V. and Singh, S.B. "New placement strategy for buffers in critical chain", In Proceedings of the Second International Conference on Soft Computing for Problem Solving (SocProS 2012), December 28-30, pp. 429-436, Springer, New Delhi (2012).

14. Van de Vonder, S., Demeulemeester, E., Herroelen, W., and Leus, R. "The use of buffers in project management: The trade-off between stability and makespan", International Journal of Production Economics, 97(2), pp. 227-240 (2005).

15. Tukel, O.I., Rom, W.O., and Eksioglu, S.D. "An investigation of buffer sizing techniques in critical chain scheduling", European Journal of Operational Research, 172(2), pp. 401-416 (2006).
16. Newbold, R.C., Project Management in the Fast Lane: Applying the Theory of Constraints, CRC Press (1998).

17. Azaron, A. and Modarres, M. "Project completion time in dynamic PERT networks with generating projects", Scientia Iranica, 14(1), pp. 56-63 (2007).

18. Leach, L.P. "Critical chain project management improves project performance", Project Management Journal, 30, pp. 39-51 (1999).

19. Zhang, J., Song, X., Chen, H., and Shi, R. "Determination of critical chain project buffer based on information flow interactions", Journal of the Operational Research Society, 67(9), pp. 1146-1157 (2016).

20. Wei, C.-C., Liu, P.-H., and Tsai, Y.-C. "Resourceconstrained project management using enhanced theory of constraint", International Journal of Project Management, 20(7), pp. 561-567 (2002).

21. Lambrechts, O., Demeulemeester, E., and Herroelen, W. "A tabu search procedure for developing robust predictive project schedules", International Journal of Production Economics, 111(2), pp. 493-508 (2008).

22. Ma, G., Li, L., and Chen, Z. "Research on the buffer sizing approach in critical chain scheduling in perspective of flexible management", in Business, Economics, Financial Sciences, and Management, Springer, pp. 61-68 (2012).

23. Truc, N.T.N., Goto, H., Takahashi, H., Yoshida, S., and Takei, Y. "Critical chain project management based on a max-plus linear representation for determining time buffers in multiple projects", Journal of Advanced Mechanical Design, Systems, and Manufacturing, 6(5), pp. 715-727 (2012).

24. Liu, D.Y., Chen, J.G., and Peng, W. "A new buffer setting method based on activity attributes in construction engineering", Applied Mechanics and Materials, 174, pp. 3274-3281 (2012).

25. Peng, W.L. and Jiao, Q. "A critical chain project scheduling problem with multi-mode" in Materials Science Forum, Trans Tech Publ. (2012).

26. Peng, W. and Huang, M. "A critical chain project scheduling method based on a differential evolution algorithm", International Journal of Production Research (ahead-of-print), pp. 1-10 (2013).

27. Ghamginzadeh, A., Najafi, A., and Azimia, P. "Solving a multi-objective resource-constrained project scheduling problem using a cuckoo optimization algorithm", Scientia Iranica, Transactions E, Industrial Engineering, 21(6), p. 2419 (2014).

28. Ma, G., Wang, A., Li, N., Gu, L., and Ai, Q. "Improved critical chain project management framework for scheduling construction projects", Journal of Construction Engineering and Management, 140(12), p. 04014055 (2014).

29. Ma, G., Gu, L., and Li, N. "Scenario-based proactive robust optimization for critical-chain project scheduling", Journal of Construction Engineering and Management, 141 (10), p. 04015030 (2015). 
30. Zhang, J., Song, X., and Díaz "Buffer sizing of critical chain based on attribute optimization", Concurrent Engineering, 22(3), pp. 253-264 (2014).

31. Bevilacqua, M., Ciarapica, F.E., Mazzuto, G., and Paciarotti, C. "Robust multi-criteria project scheduling in plant engineering and construction" In Handbook on Project Management and Scheduling, 2, pp. 1291-1305, Springer, Cham (2015).

32. Zhang, J., Song, X., and Diaz, E. "Project buffer sizing of a critical chain based on comprehensive resource tightness", European Journal of Operational Research, 248(1), pp. 174-182 (2016).

33. Iranmanesh, H., Mansourian, F., and Kouchaki, S. "Critical chain scheduling: a new approach for feeding buffer sizing", International Journal of Operational Research, 25(1), pp. 114-130 (2016).

34. Hu, X., Cui, N., Demeulemeester, E., and Bie, L. "Incorporation of activity sensitivity measures into buffer management to manage project schedule risk", European Journal of Operational Research, 249(2), pp. 717-727 (2016).

35. Hu, X., Demeulemeester, E., Cui, N., Wang, J., and Tian, W. "Improved critical chain buffer management framework considering resource costs and schedule stability", Flexible Services and Manufacturing Journal, 29(2), pp. 159-183 (2017).

36. Moder, J.J., Phillips, C.R., and Davis, E.W., Project Management with CPM, PERT, and Precedence Diagramming, Publisher New York: Van Nostrand Reinhold (1983).

37. Herroelen, W. and Leus, R. "On the merits and pitfalls of critical chain scheduling", Journal of Operations Management, 19(5), pp. 559-577 (2001).

38. Kolisch, R. and Hartmann, S. "Heuristic algorithms for the resource-constrained project scheduling problem: Classification and computational analysis", In Project Scheduling, pp. 147-178, Springer, Boston, MA (1999).

39. Xiangyuan, S. "Identifying the critical chain in the critical chain project management", In Future Wireless Networks and Information Systems, pp. 387-393, Springer, Berlin, Heidelberg (2012).

40. Hartmann, S. and Kolisch, R. "Experimental evaluation of state-of-the-art heuristics for the resourceconstrained project scheduling problem", European Journal of Operational Research, 127(2), pp. 394-407 (2000).

41. Vanhoucke, M., Critical Chain/Buer Management, in Project Management with Dynamic Scheduling, Springer, pp. 185-206 (2012)

42. Pozzi, L. "The coefficient of relative risk aversion: a Monte Carlo study investigating small sample estimator problems", Economic Modelling, 20(5), pp. 923940 (2003).

43. Kwak, Y.H. and Ingall, L. "Exploring Monte Carlo simulation applications for project management", Risk Management, 9(1), pp. 44-57 (2007).
44. Chtourou, H. and Haouari, M. "A two-stage-priorityrule-based algorithm for robust resource-constrained project scheduling", Computers \& Industrial Engineering, 55(1), pp. 183-194 (2008).

45. Roel, L. "The generation of stable project plans", Complexity and Exact Algorithms, Leuven, KU Leuven, Faculteit Economische en Toegepaste Economische Wetenschappen (2003).

46. Bie, L., Cui, N., and Zhang, X. "Buffer sizing approach with dependence assumption between activities in critical chain scheduling", International Journal of Production Research, 50(24), pp. 7343-7356 (2012).

\section{Biographies}

Ramin Ansari is a Civil Engineer and the C.E.O of a construction company which has carried out projects all over Iran. He has been conducting many studies for the Project Management of Iranian Companies for eight years, and has published more than ten national and international papers on Project Management, Scheduling, Optimization, etc. He has gained practical experience of the construction industry for more than twelve years. He has received his MS in Construction Engineering and Management field at Amirkabir University of Technology (Tehran Polytechnic). His main research fields are project and contract management. $\mathrm{He}$ is a $\mathrm{PhD}$ candidate in Construction Engineering and Management at the Iran University of Science and Technology at the present time.

Ahmad Makui is an Associate Professor of Industrial Engineering at Iran University of Science and Technology. He received his MS in 1991 and $\mathrm{PhD}$ in 1994 in Industrial Engineering at the Iran University of Science and Technology. He lectures in the field of scheduling, project and product planning and control, and MCDM. He has published three books in Persian and more than one hundred national and international papers on scheduling, etc. His main research interests consist of scheduling, MCDM, and supply chain management.

Parviz Ghoddousi is an Associate Professor of Civil Engineering at Iran University of Science and Technology. He received his $\mathrm{MS}$ and $\mathrm{PhD}$ degrees at University of Leeds in Construction Engineering in 1987 and 1992, respectively. He lectures in the field of project management, project planning and control, equipment and methods, concrete technology. $\mathrm{He}$ has published eight books in Persian and more than fifty national and international papers on construction engineering and concrete technology. His main research interests consist of project management and concrete durability. 